\title{
Lessons for reviewing clinical trials using induced pluripotent stem cells: examining the case of a first-in-human trial for age-related macular degeneration
}

\author{
Kayo Takashima*,1, Yusuke Inoue ${ }^{1}$, Shimon Tashiro² \& Kaori Muto ${ }^{1}$ \\ ${ }^{1}$ Department of Public Policy, The Institute of Medical Science, The University of Tokyo, Tokyo, Japan \\ ${ }^{2}$ Bioethics Section, Center for Public Health Sciences, National Cancer Center, Tokyo, Japan \\ * Author for correspondence: kayot-tky@umin.ac.jp
}
"when informing participants of potential direct therapeutic benefits, the committees should identify whether the participants can make reasonable choices free of therapeutic misconception."

First draft submitted: 13 September 2017; Accepted for publication: 5 October 2017; Published online: 6 December 2017

\begin{abstract}
Keywords: age-related macular degeneration $\bullet$ AMD $\bullet$ committee meeting minutes $\bullet$ direct benefit $\bullet$ first-in-human trial • induced pluripotent stem cell • IPSC • reasonable accommodation • protecting research participants • review committee $\bullet$ risk assessment $\bullet$ subject selection
\end{abstract}

\section{Background of the first-in-human trial using induced pluripotent stem cells}

In 2014, Dr Takahashi (RIKEN, Kobe, Japan) and her research team conducted the first-in-human (FIH) trial using induced pluripotent stem cells (iPSCs). In this pilot case (hereafter, this trial will be referred to as the iPSC-FIH trial), a sheet of retinal pigment epithelial (RPE) cells was transplanted into a patient. The aim of the trial was to assess the safety and adverse event profile, as well as the feasibility of transplanting a sheet of RPE cells that differentiated from autologous iPSCs into a patient with neovascular age-related macular degeneration (AMD), a degenerative retinal disease that may result in vision loss in elderly populations [1]. Along with the ambition of the Japanese government, which sought to dominate the global field of regenerative medicine [2], the news of this trial attracted great attention to diverse communities such as patients, the general public, and stem cell researchers [3]. This mounting excitement about regenerative medicine as a 'dream therapy', however, put the five committees reviewing the iPSC-FIH trial under significant stress during the review process from 2012 to 2013.

The ethical concerns of the iPSC-FIH trial have been examined [4,5], yet these analyses did not include assessments of discussions that took place in review committee meetings of this trial. Therefore, for the present study to identify future lessons for the committees reviewing cutting-edge FIH trials using novel stem cells, the minutes of the iPSC-FIH trial review committee meetings were examined.

\section{Review process for the iPSC-FIH trial}

The review process at the time of the trial was as follows:

First, institutional review boards (IRBs) of the respective institutions reviewed the trial protocol. After obtaining approval from each IRB, the proposal was submitted to the Japanese Ministry of Health, Labor and Welfare for review by the Committee for Science and Technology and its Special Committee for Human Stem Cell Clinical Research, which served as national review committees (NRCs). After the decisions of the NRCs were issued to the institutions, the IRBs then discussed the final protocol by conforming to the NRCs' decisions.

The protocol of the iPSC-FIH trial was reviewed in 14 meetings held by three IRBs and two NRCs from 5 July 2012 to 29 July 2013; the Ministry granted its approval on 19 July 2013. These meeting minutes were obtained for analysis from the relevant RIKEN and Ministry of Health, Labor and Welfare websites. The minutes from the remaining two IRBs were not available online; thus, these were requested directly from them. One NRC held an 
additional meeting to discuss the results of the whole-genome sequences and the whole-methylome analysis of the patients' fibroblasts as well as the iPSCs derived from the fibroblasts. The minutes of this additional meeting were not made available.

Two IRBs eventually approved the trial protocol with oral requests but no written conditions, while one IRB and the NRC granted approval with written conditions, such as that the results of the final safety examination be reported to each committee.

The meetings covered a wide range of subjects, including the quality control and risks of the trial (e.g., the risks of iPSCs especially the risks of tumorigenicity and genetic aberrations, contamination with harmful viruses and bacteria during the process of establishing iPSCs, and surgical complications), protection of research participants (e.g., the suitability of the patient information sheet in securing informed consent, preventing therapeutic misconception in which the research participant fails to distinguish between participating in the trial and receiving standard treatment [6], compensation for research-related health damages, and psychological care for participants), the background of the trial and target disease, and the appropriateness of the review process.

The present article highlights how the review committees evaluated the risks and benefits of the iPSC-FIH trial. Moreover, conversations regarding how information on the potential direct benefits could be provided to participants, in particular relating to how to prevent therapeutic misconception and how to assist the participants in making reasonable decisions, are of special focus in the present report.

\section{Risk assessment of tumorigenicity}

Risk assessment was a significant challenge in the iPSC-FIH trial because the associated safety concerns included risk of carcinogenesis and other unpredictable risks of harm. Furthermore, this trial was regarded as a remarkable milestone in the field of regenerative medicine. News of the trial had attracted great public attention including that of patients, the public, industry, academia, and the Japanese government, which implied that any factor leading to failure would not be tolerated. There was no doubt that each review committee experienced extreme pressure to grant an approval.

The committees requested that the researchers conduct various tests and revisions to eliminate any risks involved in the trial, especially concerning safety verification for tumorigenicity. These tests included the examination of cells that resembled mesenchymal stem cells derived from dedifferentiated RPEs, the detection of genetic aberrations and vector suitability for iPSC establishment, and treatments for potential carcinogenesis.

One NRC brought in additional professionals to evaluate the safety and quality control of the transgenesis. The NRC considered that gene transfer in the establishment of iPSCs closely resembled that of gene therapy because the established cell containing the transgene was introduced into humans [7]. This led to a discussion on the accuracy of transgenic vector selection, which did not appear in previous IRB reviews. The NRC was concerned about the use of EBNA-1 plasmid vector as the transgenic vector to establish iPSCs because of its lack of previous use in clinical settings. The NRC thus focused on the analysis of the potential risks of oncogenesis, as it was concerned that the plasmid vector would remain inside the bodies of the participants and become cancerous. One NRC member requested the use of a vector that was theoretically and clinically proven to be safe for gene therapy. In response to this cautionary request, the researchers emphasized that other vectors did not allow persistent gene expression for 2 weeks, an indispensable process for iPSC establishment, and that they had selected GMP-grade vectors for this trial. The researchers also claimed that the vector disappearance after repeated cell subculture reduced the risk of tumorigenicity. In its final decision, the NRC accepted the use of the EBNA-1 plasmid vector, provided that residual plasmids in the iPSCs be carefully detected. The NRC also requested that the whole-genome sequence and the whole-methylome analysis of the patients' cell and iPSCs be examined, and that the examination results be reported to each committee.

Regarding evaluation of other potential risks, one NRC member repeatedly insisted that an in vitro test aimed at detecting viruses be conducted due to the retina's proximity to the brain, although the researchers did plan to conduct PCR (which detects viruses in autologous cells). Ultimately, the researchers accepted this request; however, upon obtaining the NRC's recommendation, some IRB members questioned the necessity of this test for the trial.

These discussions centered on avoiding conceivable risks as much as possible; however, apart from specifying the target population to be selected, they did not appear to have substantially evaluated the favorable risk-benefit ratio of participants in clinical settings. With regards to the target population, one IRB noted that AMD was a suitable subject for the FIH trial because abnormalities were easy to detect and treat. On the other hand, another IRB stated that although the trial might improve a participant's vision, the risks to the participant were too high 
compared with the relatively small therapeutic benefits. One NRC member had also concerns about the possible occurrence of invasive teratoma at the intersection of the optic nerve projecting from the retina, where even a localized teratoma might result in the need to remove both eyes. In the end, the entire protocol was approved without any recommendation to change the target population.

\section{Informing participants of potential direct benefits}

One of the potential therapeutic benefits of this trial was projected to be the (partial) restoration of a patient's eyesight. The researchers expected that a visual acuity of 0.06 or 0.05 might be increased to 0.1 , although this depended on the condition of the participant's retina; the researches expected, at the very least, for retina sensitivity to increase. The discussion here focused on how to inform participants of potential yet unproven direct medical benefits such as the possible restoration of eyesight. Dr Takahashi expressed concerns about therapeutic misconceptions held by patients and the public, owing to society's high expectations of regenerative medicine. (In the meeting with patient groups, for example, she carefully explained that the trial was an early phase trial to avoid unrealistic expectations [8]. She also set up meetings with the media to promote a common understanding).

The researchers appeared to be confused by the contradictory opinions of the committees as to whether the researchers should explain the anticipated therapeutic benefits of the trial to potential participants. One IRB advised the researchers against describing the trial's potential direct benefits in the patient information sheets, as the primary end point of the trial was to evaluate safety. In contrast, another IRB argued that patients might be unwilling to participate if they were informed of only a 'slight improvement' in vision. In the end, the IRBs accepted the researchers' proposal to describe the potential therapeutic benefits of the trial as being less than what was in fact expected.

Regarding therapeutic misconception, the IRBs and one NRC also discussed how to approach the issue of press briefings and media reports, and expressed concern that patients might take media interviews voluntarily without first discussing with the research team and/or the institutions. If this were to occur, a patient's positive review, based on initial temporary results, might lead to the incorrect belief that even the early phase trial could result in a cure for AMD. The IRB members discussed how to convey news reports of the study's results to patients and the public in advance of such occurrences. The researchers mentioned that news reports had generated high expectations among many patients who wanted to participate in the trial; thus, the research team decided to choose only those candidates who fully understood the trial's aim.

\section{Potential suggestions for future review committees}

\section{Mandatory test or desirable test}

As this trial involved various advanced technologies, each expert argued over the best method of risk evaluation based on his or her professional perspective (e.g., on vector selection and in vitro testing as mentioned previously). After receiving the final opinion of the NRCs, one IRB member noted that the risk evaluation tests could be divided into three categories: mandatory tests, limited requirement tests for this trial only, and desirable tests. One of the suggestions arising from the present analysis of the discussion is that future review committees should identify whether their requirements for risk evaluation tests are mandatory for the trial or simply desirable for the purpose of gaining scientific knowledge. Furthermore, if the risk assessments are based solely on 'precautionary principles', the clinical trials, especially the FIH trials, will likely fail to receive approval because no committee should approve a trial without sufficient knowledge of its risks [9].

The International Society for Stem Cell Research Guidelines recommend a 'systematic review of available scientific evidence' [10]. This recommendation is reasonable; however, a possible question arising for the iPSC-FIH trial would be how to define 'available scientific evidence'. Moreover, according to Kimmelman's theory, optimism bias by researchers is a major concern in translational research, and translational trials should always be maintained within 'modest translational distance' [11]. The question then becomes whether the excessive pessimism bias of the review committee is also within suitable translational distance.

Further, it might become impossible to conduct trials if there are many requirements of 'desirable tests'. The iPSCFIH trial was already subjected to various requirements set by the committees, including the timeline requirements of funding agencies. That the researchers were able to endure these requirements may have been largely thanks to the generous financial support provided by the Japanese government (in line with its national strategy to improve and facilitate the field of regenerative medicine) [2] and the technical support of many researchers in related fields. 
The first suggestion to future review committees is to identify mandatory tests for clinical application when requiring risk evaluation tests in FIH trials. A zero-risk status may be ideal for conducting FIH trials; however, if there are many requirements that do not exactly relate to clinical applications, they might make clinical applications impossible or exhaust the trial's budget.

\section{Respecting the participants' reasonable choice}

Informing potential participants of anticipated direct medical benefits might result in a conflict between the need to prevent therapeutic misconceptions and the need to provide accurate information that supports reasonable decision making by potential participants. The researchers in this trial sought to inform their patients of the potential direct medical benefits in a manner that would avoid overly high expectations. The researchers and committees appeared to have tried to prevent such misconceptions at both the individual level and the collective level [12].

In terms of collective therapeutic misconceptions, because of the media's positive coverage of regenerative medicine research similar to that in the genetic research field [13], the patient community and the public had high expectations of the trial as an efficacious therapy, even during its early phase trial for safety evaluation. To address these expectations, Dr Takahashi stated on many occasions that "our achievement can be compared with the Wright Brothers' early successful flight of just 300 meters (in 1903)" [14] to clarify how challenging and distant an FIH trial was from its application as a standard treatment.

To manage expectations, it might be possible to provide information on the potential harm of the FIH trials and referred to potential therapeutic benefits as having a 'reasonable chance', based on preclinical analyses; this might have helped the decision making of the participants toward 'reasonable choices' [15]. According to King's argument, uncertainty exists equally for both risk and benefit [15]; thus, it is crucial to inform participants of both the risks and benefits in a detailed and accurate manner.

The second suggestion to future committees is that, when informing participants of potential direct therapeutic benefits, the committees should identify whether the participants can make reasonable choices free of therapeutic misconception. When discussing these matters, the committees should also recognize the researchers' efforts to prevent therapeutic misconception based on social responses to the trial.

\section{Considering reasonable accommodations for participants}

'Reasonable accommodations' refer to necessary and appropriate modifications and adjustments, which do not impose a disproportionate or undue burden that ensure the enjoyment or exercise of all human rights and fundamental freedoms by individuals with disabilities on an equal basis with others [16]. For participants with ophthalmological diseases, the provision of audio-recorded material could improve their understanding of the trial, reduce feelings of unease, help avoid therapeutic misconception, and support their decision making. The research team included this information as a one-page version of their website with a text-to-speech friendly interface [17], though no committee discussed the provision of special assistance to participants with AMD.

In such a cutting-edge FIH trial, it is understandable that the focus of the review committees was on whether the use of novel types of cells was safe. However, the committees should have discussed the characteristics of the target disease and the actual needs of the participants. The welfare of the participants should have been reviewed by considering the condition of the target disease and assessing whether the patients would require special assistance in order to better understand information regarding the trial.

\section{Future perspective}

With the rapid growth in the field of human stem cell research, the existing review systems have been revised, and discussions regarding the safety evaluation systems for regenerative medicine have advanced in regulatory science. The review process of human stem cell clinical research in Japan is now governed by the Act on the Safety of Regenerative Medicine, which was introduced in 2014, or by the Pharmaceutical and Medical Device Act, which was revised in 2014. In the USA, the 21st Century Cures Act enacted in 2016 describes a new category of "regenerative medicine advanced therapies". Following such examples, other countries conducting trials with stem cell therapy may also revise their regulations concerning stem cell research to suit their present needs.

When such regulatory systems are revised, perspectives on protecting research participants should also be reconsidered given the nature of FIH trials. Scientists are currently planning FIH trials using iPSCs for various diseases, including corneal diseases, Parkinson's disease and severe heart failure. It is hoped that the recommendations made in this paper - namely, to set appropriate lines for risk assessment, support reasonable participant decision 
making free of therapeutic misconception, and consider participant care based on the characteristics of the target disease - will be accounted for during the review processes of these trials. Although careful attention has been paid to the specificity of FIH trials, reviewers must, as a rule, also discuss the universal viewpoint of protecting the rights and welfare of research participants.

This paper analyzed only one review process regarding FIH trials using iPSCs. If various case studies on the review processes of FIH stem cell trials or other cutting-edge biotherapeutic trials from around the world were similarly discussed, such case studies would contribute to establishing or improving guidelines for review committees to further improve the quality of these discussions.

\section{Acknowledgements}

The authors extend special thanks to the Translational Research Ethics Committee of RIKEN, and both the Committee for Science and Technology of the Health Science Council and the Health Science Council of the Health and Stem Cell Research Oversight Committee of the Ministry of Health, Labor and Welfare for making the relevant meeting minutes available on their websites. The authors are especially grateful to the Institutional Committee for Regenerative Medicine of the Institute of Biomedical Research and Innovation, and the Research Ethics Committee of the Kobe City Medical Center General Hospital for providing the meeting minutes and permitting their use in this analysis.

\section{Financial \& competing interests disclosure}

This research was supported by the Highway Program for Realization of Regenerative Medicine from the Japan Agency for Medical Research and Development (AMED). The authors have no other relevant affiliations or financial involvement with any organization or entity with a financial interest in or financial conflict with the subject matter or materials discussed in the manuscript apart from those disclosed.

Funded writing assistance (English editing service) for this manuscript was supported by AMED.

\section{Open access}

This work is licensed under the Attribution-NonCommercial-NoDerivatives 4.0 Unported License. To view a copy of this license, visit http://creativecommons.org/licenses/by-nc-nd/4.0/

\section{References}

1. Mandai M, Watanabe A, Kurimoto Y et al. Autologous induced stem-cell-derived retinal cells for macular degeneration. N. Engl. J. Med. 376(11), 1038-1046 (2017).

2. Fox C. Historic Japan stem cell trial approved. Bioscience Technology Online (2013). www.biosciencetechnology.com/article/2013/08/historic-japan-stem-cell-trial-approved

3. Reardon S, Cyranoski D. Japan stem-cell trial stirs envy - researchers elsewhere can't wait to test iPS cells in humans. Nature News (2014). www.nature.com/news/japan-stem-cell-trial-stirs-envy-1.15935\#auth-1

4. Habets MG, van Delden JJ, Bredenoord AL. The inherent ethical challenge of first-in-human pluripotent stem cell trials. Regen. Med. 9(1), 1-3 (2014).

5. Nakano-Okuno M, Borah BR, Nakano I. Ethics of iPSC-based clinical research for age-related macular degeneration: patient-centered risk-benefit analysis. Stem Cell Rev. Rep. 10(6), 743-752 (2014).

6. Lidz CW, Albert K, Appelbaum P,Dunn LB, Overton E, Pivovarova E., Why is therapeutic misconception so prevalent? Camb. Q. Healthc. Ethics 24(2), 231-241 (2015).

7. Committee for Science and Technology, the Ministry of Health, Labor, and Welfare. Handling of clinical research on age-related macular degeneration using iPS cells. Document 4 (2012). www.mhlw.go.jp/stf/shingi/2r9852000002qw9s-att/2r9852000002r3am.pdf (In Japanese).

8. Takahashi M, Muto K. Collaboration in clinical research implementation: discussion between patients and researcher Japanese Retinitis Pigmentosa Society and Department of Public Policy, The Institute of Medical Science, The University of Tokyo (2014). (Accessed 20 August 2017) www.pubpoli-imsut.jp/files/files/15/0000015.pdf

9. Peterson M. The precautionary principle should not be used as a basis for decision-making. EMBO Rep. 8(4), 305-308 (2007).

10. International Society for Stem Cell Research. Guidelines for Stem Cell Research and Clinical Translation (2016). www.isscr.org/docs/def ault-source/all-isscr-guidelines/guidelines-2016/isscr-guidelines-for-stem-cell-research-and-clinical-translationd 67119731dff6ddbb37cff0000940c19.pdf?sfvrsn=4

11. Kimmelman J. Gene Transfer and the Ethics of First-in-Human Research: Lost in Translation. Cambridge University Press, NY, USA, 1-205 (2009).

12. Dresser R. When Science Offers Salvation: Patient Advocacy and Research Ethics. Oxford University Press, NY, USA, 8-15 (2002). 
13. Benjaminy S, Kowal SP, MacDonald IM, Bubela T. Communicating the promise for ocular gene therapies: challenges and recommendations. Am. J. Ophthalmol. 160(3), 408-415 (2015).

14. iPS researcher: epoch-making clinical test clears only first step. (2014). www.japanbullet.com/news/ips-researcher-epoch-making-clinical-test-clears-only-first-step

15. King NMP. Defining and describing benefit appropriately in clinical trials. J. Law Med. Ethics 28(4), 332-343 (2000).

16. United Nations. Convention on the rights of persons with disabilities. (2006). https://treaties.un.org/Pages/ViewDetails.aspx?src=IND\&mtdsg_no=IV-15\&chapter=4\&lang=en

17. Riken and Foundation for Biomedical Research and Innovation. Pilot safety study of iPSC-based intervention for wet-type AMD. www.riken-ibri.jp/AMD/english/index.html 